\title{
Gronwall-Bellman Type Inequalities and Their Applications to Fractional Differential Equations
}

\author{
Jing Shao ${ }^{1,2}$ and Fanwei Meng ${ }^{1}$ \\ ${ }^{1}$ School of Mathematical Sciences, Qufu Normal University, Qufu, Shandong 273165, China \\ ${ }^{2}$ Department of Mathematics, Jining University, Qufu, Shandong 273155, China \\ Correspondence should be addressed to Jing Shao; shaojing99500@163.com
}

Received 29 May 2013; Accepted 22 July 2013

Academic Editor: Irena Lasiecka

Copyright (c) 2013 J. Shao and F. Meng. This is an open access article distributed under the Creative Commons Attribution License, which permits unrestricted use, distribution, and reproduction in any medium, provided the original work is properly cited.

Some new weakly singular integral inequalities of Gronwall-Bellman type are established, which can be used in the qualitative analysis of the solutions to certain fractional differential equations.

\section{Introduction}

Gronwall-Bellman type integral inequalities play increasingly important roles in the study of quantitative properties of solutions of differential and integral equations, as well as in the modeling of engineering and science problems. The integrals concerning this type of inequalities have regular or continuous kernels, but some problems of theory and practicality require us to solve integral inequalities with singular kernels; see $[1-4]$ and the references cited therein. For example, Ye and Gao [5] considered the integral inequalities of HenryGronwall type and their applications to fractional differential equations with delay; Ma and Pečarić [4] established some weakly singular integral inequalities of Gronwall-Bellman type and used them in the analysis of various problems in the theory of certain classes of differential equations, integral equations, and evolution equations.

In this paper, we study a certain class of nonlinear inequalities of Gronwall-Bellman type, which generalizes some known results and can be used as handy and effective tools in the study of differential equations and integral equations. Furthermore, applications of our results to fractional differential are also involved.

\section{Preliminary Knowledge}

In this section, we give some inequalities, which will be used in the proof of the main results.
Lemma 1 (Jensen's inequality). Let $n \in N$, and let $a_{1}, \ldots, a_{n}$ be nonnegative real numbers. Then, for $r>1$,

$$
\left(\sum_{i=1}^{n} a_{i}\right)^{r} \leq n^{r-1} \sum_{i=1}^{n} a_{i}^{r}
$$

Lemma 2. Let $I=\left[t_{0}, T\right) \subset \mathbf{R}, k(t), b(t), p(t) \in C\left(I, \mathbf{R}^{+}\right)$, $\left(\mathbf{R}^{+}=[0, \infty), T \leq \infty\right)$. If $u(t) \in C\left(I, \mathbf{R}^{+}\right)$, and

$$
u(t) \leq k(t)+\int_{t_{0}}^{t} b(s) u(s) d s+\int_{t_{0}}^{t} p(s) u^{\gamma}(s) d s, \quad t \in I,
$$

where $0 \leq \gamma<1$. Then, for $t \in I$, one has

$$
\begin{aligned}
u(t) \leq & {\left[A^{1-\gamma}(t)+(1-\gamma) \int_{t_{0}}^{t} e^{(\gamma-1) \int_{t_{0}}^{s} b(\sigma) d \sigma} p(s) d s\right]^{1 /(1-\gamma)} } \\
& \times e^{\int_{t_{0}}^{t} b(s) d s},
\end{aligned}
$$

where $A(t)=\max _{t_{0} \leq s \leq t}\{k(t)\}$.

Proof. Given $t_{0}<T_{0}<T$, for $t \in\left[t_{0}, T_{0}\right]$,

$$
u(t) \leq A\left(T_{0}\right)+\int_{t_{0}}^{t} b(s) u(s) d s+\int_{t_{0}}^{t} p(s) u^{\gamma}(s) d s .
$$


Define a function $z(t)=A\left(T_{0}\right)+\int_{t_{0}}^{t} b(s) u(s) d s+\int_{t_{0}}^{t} p(s)$ $u^{\gamma}(s) d s, t \in\left[t_{0}, T_{0}\right]$; then $z\left(t_{0}\right)=A\left(T_{0}\right), u(t) \leq z(t), z(t)$ is positive and nondecreasing for $t \in I$, and

$$
z^{\prime}(t)=b(t) u(t)+p(t) u^{\gamma}(t) \leq b(t) z(t)+p(t) z^{\gamma}(t) .
$$

Let $z(t)=C(t) e^{\int_{t_{0}}^{t} b(s) d s}$, and $C\left(t_{0}\right)=z\left(t_{0}\right)=A\left(T_{0}\right)$; we obtain $C(t) \leq\left[A^{1-\gamma}\left(T_{0}\right)+(1-\gamma) \int_{t_{0}}^{t} e^{(\gamma-1) \int_{t_{0}}^{s} b(\sigma) d \sigma} p(s) d s\right]^{1 /(1-\gamma)}$,

which implies that

$$
\begin{aligned}
z(t) \leq & {\left[A^{1-\gamma}\left(T_{0}\right)+(1-\gamma) \int_{t_{0}}^{t} e^{(\gamma-1) \int_{t_{0}}^{s} b(\sigma) d \sigma} p(s) d s\right]^{1 /(1-\gamma)} } \\
& \times e^{\int_{t_{0}}^{t} b(s) d s}, \quad t \in\left[t_{0}, T_{0}\right] .
\end{aligned}
$$

And so

$$
\begin{aligned}
u\left(T_{0}\right) \leq & {\left[A^{1-\gamma}\left(T_{0}\right)+(1-\gamma) \int_{t_{0}}^{T_{0}} e^{(\gamma-1) \int_{t_{0}}^{s} b(\sigma) d \sigma} p(s) d s\right]^{1 /(1-\gamma)} } \\
& \times e^{\int_{t_{0}}^{T_{0}} b(s) d s}, \quad t \in\left[t_{0}, T_{0}\right] .
\end{aligned}
$$

By the arbitrary of $T_{0} \in I$, we obtain the inequality (3). The proof is complete.

Lemma 3. Let $k(t), b(t), p(t) \in C\left(I, \mathbf{R}^{+}\right), \varphi(t) \in C\left(\left[t_{0}-\right.\right.$ $\left.\left.r, t_{0}\right], \mathbf{R}^{+}\right)$, and $k\left(t_{0}\right)=\varphi\left(t_{0}\right)$. If $u(t) \in C\left(I, \mathbf{R}^{+}\right)$and

$$
\begin{gathered}
u(t) \leq k(t)+\int_{t_{0}}^{t} b(s) u(s) d s+\int_{t_{0}}^{t} p(s) u^{\gamma}(s-r) d s, \\
t \in I,
\end{gathered}
$$

where $0 \leq \gamma<1$ and $r \geq 0$ is a real constant, then

$$
\begin{aligned}
u(t) \leq & {\left[A^{1-\gamma}(t)+(1-\gamma) \int_{t_{0}}^{t} e^{(\gamma-1) \int_{t_{0}}^{s} b(\sigma) d \sigma} p(s) d s\right]^{1 /(1-\gamma)} } \\
& \times e^{\int_{t_{0}}^{t} b(s) d s}, \quad t \in\left[t_{0}+r, T\right) \\
u(t) \leq & {\left[A\left(t_{0}+r\right)+\int_{t_{0}}^{t_{0}+r} p(s) \varphi^{\gamma}(s-r) d s\right] } \\
& \times e^{\int_{t_{0}}^{t} b(s) d s}, \quad t \in\left[t_{0}, t_{0}+r\right)
\end{aligned}
$$

where $A(t)$ is defined as that in Lemma 2.
Proof. For $t \in\left[t_{0}, t_{0}+r\right]$, we have

$$
\begin{aligned}
u(t) \leq & k(t)+\int_{t_{0}}^{t} b(s) u(s) d s+\int_{t_{0}}^{t} p(s) \varphi^{\gamma}(s-r) d s \\
\leq & A\left(t_{0}+r\right)+\int_{t_{0}}^{t} b(s) u(s) d s \\
& +\int_{t_{0}}^{t_{0}+r} p(s) \varphi^{\gamma}(s-r) d s .
\end{aligned}
$$

By Gronwall inequality, we have the inequality (11). We prove that (10) holds for $t \in\left[t_{0}+r, T\right)$ now. Given that $T_{0} \in\left[t_{0}+r, T\right)$ and for $t \in\left[t_{0}+r, T_{0}\right]$, we get

$$
u(t) \leq A\left(T_{0}\right)+\int_{t_{0}}^{t} b(s) u(s) d s+\int_{t_{0}}^{t} p(s) u^{\gamma}(s-r) d s .
$$

Define a function $z(t)=A\left(T_{0}\right)+\int_{t_{0}}^{t} b(s) u(s) d s+\int_{t_{0}}^{t} p(s) u^{\gamma}(s-$ $r) d s, t \in\left[t_{0}+r, T_{0}\right]$; then $z\left(t_{0}\right)=A\left(T_{0}\right), u(t) \leq z(t), z(t)$ is positive and nondecreasing for $t \in\left[t_{0}+r, T_{0}\right]$, and

$$
\begin{aligned}
z^{\prime}(t) & =b(t) u(t)+p(t) u^{\gamma}(t-r) \\
& \leq b(t) z(t)+p(t) z^{\gamma}(t-r) \\
& \leq b(t) z(t)+p(t) z^{\gamma}(t) .
\end{aligned}
$$

As that in the proof of Lemma 2, we obtain

$$
\begin{aligned}
z(t) \leq & {\left[A^{1-\gamma}\left(T_{0}\right)+(1-\gamma) \int_{t_{0}}^{t} e^{(\gamma-1) \int_{t_{0}}^{s} b(\sigma) d \sigma} p(s) d s\right]^{1 /(1-\gamma)} } \\
& \times e^{\int_{t_{0}}^{t} b(s) d s}, \quad t \in\left[t_{0}+r, T_{0}\right] .
\end{aligned}
$$

And then

$$
\begin{aligned}
u\left(T_{0}\right) \leq & {\left[A^{1-\gamma}\left(T_{0}\right)+(1-\gamma) \int_{t_{0}}^{T_{0}} e^{(\gamma-1) \int_{t_{0}}^{s} b(\sigma) d \sigma} p(s) d s\right]^{1 /(1-\gamma)} } \\
& \times e^{\int_{t_{0}}^{T_{0}} b(s) d s}, \quad t \in\left[t_{0}+r, T_{0}\right] .
\end{aligned}
$$

By the arbitrary of $T_{0} \in\left(t_{0}+r, T\right)$, we obtain the inequality (10). The proof is complete.

\section{Main Results}

Now, we are in a position to deal with the integral inequality with weak singular kernels.

Theorem 4. Let $a(t), b(t), p(t) \in C\left(I, \mathbf{R}^{+}\right)$. If $u(t) \in C\left(I, \mathbf{R}^{+}\right)$ and

$$
\begin{aligned}
u(t) \leq & a(t)+\int_{t_{0}}^{t}(t-s)^{\beta-1} b(s) u(s) d s \\
& +\int_{t_{0}}^{t}(t-s)^{\beta-1} p(s) u^{\gamma}(s) d s, \quad t \in I,
\end{aligned}
$$


where $\beta>0$ and $0<\gamma<1$ are constants, then the following assertions hold.

(i) Suppose that $\beta>1 / 2$. Then

$$
\begin{aligned}
u(t) \leq & {\left[A_{1}^{1-\gamma}(t)+(1-\gamma) K_{1}\right.} \\
& \left.\quad \times \int_{t_{0}}^{t} e^{(\gamma-1) K_{1} \int_{t_{0}}^{s} b^{2}(\sigma) d \sigma} p^{2}(s) R_{1}(s) d s\right]^{1 / 2(1-\gamma)} \\
& \times e^{\left(t+\left(K_{1} / 2\right) \int_{t_{0}}^{t} b^{2}(s) d s\right)}, \quad t \in I
\end{aligned}
$$

where $A_{1}(t)=\max _{t_{0} \leq s \leq t}\left\{2 e^{-2 t} a^{2}(t)\right\}, K_{1}=2 \Gamma(2 \beta-$ $1) / 4^{\beta-1}$, and $R_{1}(t)=e^{2(\gamma-1) t}$.

(ii) Suppose that $\beta \in(0,1 / 2], q=(1+\beta) / \beta$, and $p=1+\beta$. Then

$u(t) \leq\left[A_{2}^{1-\gamma}(t)+(1-\gamma) K_{2}\right.$

$$
\begin{aligned}
& \left.\times \int_{t_{0}}^{t} e^{(\gamma-1) K_{2} \int_{t_{0}}^{s} b^{q}(\sigma) d \sigma} p^{q}(s) R_{2}(s) d s\right]^{1 / q(1-\gamma)} \\
\times & e^{\left(t+\left(K_{2} / q\right) \int_{t_{0}}^{t} b^{q}(s) d s\right)}, \quad t \in I,
\end{aligned}
$$

where $A_{2}(t)=\max _{t_{0} \leq s \leq t}\left\{2^{q-1} e^{-t q} a^{q}(t)\right\}, K_{2}=$ $2^{2 q-2}\left(\Gamma(1-(1-\beta) p) / p^{1-(1-\beta) p}\right)^{q / p}$ and $R_{2}(t)=$ $e^{q(\gamma-1) t}$.

Proof. (i) Using the Cauchy-Schwarz inequality, we obtain

$$
\begin{aligned}
u(t) \leq & a(t)+\left(\int_{t_{0}}^{t}(t-s)^{2 \beta-2} e^{2 s} d s\right)^{1 / 2} \\
& \times\left(\int_{t_{0}}^{t} b^{2}(s) e^{-2 s} u^{2}(s) d s\right)^{1 / 2} \\
& +\left(\int_{t_{0}}^{t}(t-s)^{2 \beta-2} e^{2 s} d s\right)^{1 / 2} \\
& \times\left(\int_{t_{0}}^{t} p^{2}(s) e^{-2 s} u^{2 \gamma}(s) d s\right)^{1 / 2} \\
\leq & a(t)+\left(\frac{2 e^{2 t} \Gamma(2 \beta-1)}{4^{\beta}}\right)^{1 / 2} \\
& \times\left(\int_{t_{0}}^{t} b^{2}(s) e^{-2 s} u^{2}(s) d s\right)^{1 / 2} \\
& +\left(\frac{2 e^{2 t} \Gamma(2 \beta-1)}{4 \beta}\right)^{1 / 2} \\
& \times\left(\int_{t_{0}}^{t} p^{2}(s) e^{-2 s} u^{2 \gamma}(s) d s\right)^{1 / 2} .
\end{aligned}
$$

Using Lemma 1, we obtain

$$
\begin{aligned}
u^{2}(t) \leq & 2 a^{2}(t)+\frac{2 e^{2 t} \Gamma(2 \beta-1)}{4^{\beta-1}} \\
& \times\left(\int_{t_{0}}^{t} b^{2}(s) e^{-2 s} u^{2}(s) d s+\int_{t_{0}}^{t} p^{2}(s) e^{-2 s} u^{2 \gamma}(s) d s\right) .
\end{aligned}
$$

Let $v(t)=\left[e^{-t} u(t)\right]^{2}$; we get

$$
\begin{aligned}
v(t) \leq & A_{1}(t)+K_{1} \int_{t_{0}}^{t} b^{2}(s) v(s) d s \\
& +K_{1} \int_{t_{0}}^{t} p^{2}(s) R_{1}(s) v^{\gamma}(s) d s .
\end{aligned}
$$

Using Lemma 2 and noticing that $A_{1}(t)$ is nondecreasing, we get

$$
\begin{aligned}
v(t) \leq & {\left[A_{1}^{1-\gamma}(t)+(1-\gamma) K_{1}\right.} \\
& \left.\quad \times \int_{t_{0}}^{t} e^{(\gamma-1) K_{1} \int_{t_{0}}^{s} b^{2}(\sigma) d \sigma} p^{2}(s) R_{1}(s) d s\right]^{1 /(1-\gamma)} \\
& \times e^{K_{1} \int_{t_{0}}^{t} b^{2}(s) d s}
\end{aligned}
$$

by the relationship of $v(t)$ and $u(t)$, the first inequality (18) holds.

(ii) By the hypothesis, we get $(1 / p)+(1 / q)=1$. Using Hölder inequality, we obtain

$$
\begin{aligned}
u(t) \leq & a(t)+\left(\int_{t_{0}}^{t}(t-s)^{p \beta-p} e^{p s} d s\right)^{1 / p} \\
& \times\left(\int_{t_{0}}^{t} b^{q}(s) e^{-q s} u^{q}(s) d s\right)^{1 / q} \\
& +\left(\int_{t_{0}}^{t}(t-s)^{p \beta-p} e^{p s} d s\right)^{1 / p} \\
& \times\left(\int_{t_{0}}^{t} p^{q}(s) e^{-q s} u^{q \gamma}(s) d s\right)^{1 / q} \\
\leq & a(t)+\left(\frac{e^{p t} \Gamma(1-(1-\beta) p)}{p^{1-(1-\beta) p}}\right)^{1 / p} \\
& \times\left(\int_{t_{0}}^{t} b^{q}(s) e^{-q s} u^{q}(s) d s\right)^{1 / q} \\
& +\left(\frac{e^{p t} \Gamma(1-(1-\beta) p)}{p^{1-(1-\beta) p}}\right)^{1 / p} \\
& \times\left(\int_{t_{0}}^{t} p^{q}(s) e^{-q s} u^{q \gamma}(s) d s\right)^{1 / q} .
\end{aligned}
$$


Using Lemma 1, we obtain

$$
\begin{aligned}
u^{q}(t) \leq & 2^{q-1} a^{q}(t)+2^{2 q-2}\left(\frac{e^{p t} \Gamma(1-(1-\beta) p)}{p^{1-(1-\beta) p}}\right)^{q / p} \\
& \times\left(\int_{t_{0}}^{t} b^{q}(s) e^{-q s} u^{q}(s) d s+\int_{t_{0}}^{t} p^{q}(s) e^{-q s} u^{q \gamma}(s) d s\right) .
\end{aligned}
$$

Let $v(t)=\left[e^{-t} u(t)\right]^{q}$, we get

$$
\begin{aligned}
v(t) \leq & A_{2}(t)+K_{2} \int_{t_{0}}^{t} b^{q}(s) v(s) d s \\
& +K_{2} \int_{t_{0}}^{t} p^{q}(s) R_{2}(s) v^{\gamma}(s) d s .
\end{aligned}
$$

Using Lemma 2 and noticing that $A_{2}(t)$ is nondecreasing, we get

$$
\begin{aligned}
v(t) \leq & {\left[A_{2}^{1-\gamma}(t)+(1-\gamma) K_{2}\right.} \\
& \left.\quad \times \int_{t_{0}}^{t} e^{(\gamma-1) K_{2} \int_{t_{0}}^{s} b^{q}(\sigma) d \sigma} p^{q}(s) R_{2}(s) d s\right]^{1 / q(1-\gamma)} \\
& \times e^{K_{2} \int_{t_{0}}^{t} b^{q}(s) d s}
\end{aligned}
$$

and by the relation of $u(t)$ and $v(t),(19)$ holds. The proof is complete.

Theorem 5. Let $a(t), b(t), p(t) \in C\left(I, \mathbf{R}^{+}\right)$, and $a\left(t_{0}\right)=\varphi\left(t_{0}\right)$. If $u(t) \in C\left(\left[t_{0}-r, T\right), \mathbf{R}^{+}\right)$with

$$
\begin{aligned}
u(t) \leq & a(t)+\int_{t_{0}}^{t}(t-s)^{\beta-1} b(s) u(s) d s \\
& +\int_{t_{0}}^{t}(t-s)^{\beta-1} p(s) u^{\gamma}(s-r) d s, \quad t \in\left[t_{0}, T\right), \\
& u(t) \leq \varphi(t), \quad t \in\left[t_{0}-r, t_{0}\right)
\end{aligned}
$$

where $\beta>0$ and $0<\gamma<1$ are constants, then the following assertions hold.

(i) Suppose that $\beta>1 / 2$. Then

$$
\begin{aligned}
u(t) \leq & {\left[A_{1}^{1-\gamma}(t)+(1-\gamma) K_{1}\right.} \\
& \left.\quad \times \int_{t_{0}}^{t} e^{(\gamma-1) K_{1} \int_{t_{0}}^{s} b^{2}(\sigma) d \sigma} p^{2}(s) R_{1}(s) d s\right]^{1 / 2(1-\gamma)} \\
& \times e^{\left(t+\left(K_{1} / 2\right) \int_{t_{0}}^{t} b^{2}(s) d s\right)}, \quad t \in\left[t_{0}+r, T\right)
\end{aligned}
$$

$$
\begin{aligned}
u(t) \leq & {\left[A_{1}\left(t_{0}+r\right)+K_{1}\right.} \\
& \left.\quad \times \int_{t_{0}}^{t_{0}+r} p^{2}(s) R_{1}(s) \varphi^{2 \gamma}(s-r) e^{-2 \gamma(s-r)} d s\right]^{1 / 2} \\
& \times e^{\left(t+\left(K_{1} / 2\right) \int_{t_{0}}^{t} b^{2}(s) d s\right)}, \quad t \in\left[t_{0}, t_{0}+r\right)
\end{aligned}
$$

where $A_{1}(t), K_{1}$, and $R_{1}(s)$ are defined as those in Theorem 4.

(ii) Suppose that $\beta \in(0,1 / 2], q=(1+\beta) / \beta$, and $p=1+\beta$. Then,

$$
\begin{aligned}
u(t) \leq & {\left[A_{2}^{1-\gamma}(t)+(1-\gamma) K_{2}\right.} \\
& \left.\quad \times \int_{t_{0}}^{t} e^{(\gamma-1) K_{2} \int_{t_{0}}^{s} b^{q}(\sigma) d \sigma} p^{q}(s) R_{2}(s) d s\right]^{1 / q(1-\gamma)} \\
& \times e^{\left(t+\left(K_{2} / q\right) \int_{t_{0}}^{t} b^{q}(s) d s\right)}, \quad t \in\left[t_{0}+r, T\right), \\
u(t) \leq & {\left[A_{2}\left(t_{0}+r\right)+K_{2}\right.} \\
& \left.\quad \times \int_{t_{0}}^{t_{0}+r} p^{q}(s) R_{2}(s) \varphi^{q \gamma}(s-r) e^{-q \gamma(s-r)} d s\right]^{1 / q} \\
& \times e^{\left(t+\left(K_{2} / q\right) \int_{t_{0}}^{t} b^{q}(s) d s\right)}, \quad t \in\left[t_{0}, t_{0}+r\right),
\end{aligned}
$$

where $A_{2}(t), K_{2}$, and $R_{2}(s)$ are defined as those in Theorem 4 .

Proof. (i) Using the Cauchy-Schwarz inequality by (28), we obtain

$$
\begin{aligned}
u(t) \leq & a(t)+\left(\int_{t_{0}}^{t}(t-s)^{2 \beta-2} e^{2 s} d s\right)^{1 / 2} \\
& \times\left(\int_{t_{0}}^{t} b^{2}(s) e^{-2 s} u^{2}(s) d s\right)^{1 / 2} \\
& +\left(\int_{t_{0}}^{t}(t-s)^{2 \beta-2} e^{2 s} d s\right)^{1 / 2} \\
& \times\left(\int_{t_{0}}^{t} p^{2}(s) e^{-2 s} u^{2 \gamma}(s-r) d s\right)^{1 / 2} \\
\leq & a(t)+\left(\frac{2 e^{2 t} \Gamma(2 \beta-1)}{4^{\beta}}\right)^{1 / 2} \\
& \times\left(\int_{t_{0}}^{t} b^{2}(s) e^{-2 s} u^{2}(s) d s\right)^{1 / 2}
\end{aligned}
$$




$$
\begin{aligned}
& +\left(\frac{2 e^{2 t} \Gamma(2 \beta-1)}{4^{\beta}}\right)^{1 / 2} \\
& \times\left(\int_{t_{0}}^{t} p^{2}(s) e^{-2 s} u^{2 \gamma}(s-r) d s\right)^{1 / 2}, \quad t \in I .
\end{aligned}
$$

Using Lemma 1, we obtain

$$
\begin{aligned}
u^{2}(t) \leq & 2 a^{2}(t)+\frac{2 e^{2 t} \Gamma(2 \beta-1)}{4^{\beta-1}} \\
& \times\left(\int_{t_{0}}^{t} b^{2}(s) e^{-2 s} u^{2}(s) d s\right. \\
& \left.+\int_{t_{0}}^{t} p^{2}(s) e^{-2 s} u^{2 \gamma}(s-r) d s\right) .
\end{aligned}
$$

Let $v(t)=\left[e^{-t} u(t)\right]^{2}$, we get

$$
\begin{aligned}
v(t) \leq & A_{1}(t)+K_{1} \int_{t_{0}}^{t} b^{2}(s) v(s) d s \\
& +K_{1} \int_{t_{0}}^{t} p^{2}(s) R_{1}(s) v^{\gamma}(s-r) d s, \quad t \in I .
\end{aligned}
$$

Using Lemma 3, we get the first inequality of (29) and the second inequality of (29) is easily obtained.

(ii) By the hypothesis, we get $(1 / p)+(1 / q)=1$. Using Hölder inequality, we obtain

$$
\begin{aligned}
u(t) \leq & a(t)+\left(\int_{t_{0}}^{t}(t-s)^{p \beta-p} e^{p s} d s\right)^{1 / p} \\
& \times\left(\int_{t_{0}}^{t} b^{q}(s) e^{-q s} u^{q}(s) d s\right)^{1 / q} \\
& +\left(\int_{t_{0}}^{t}(t-s)^{p \beta-p} e^{p s} d s\right)^{1 / p} \\
& \times\left(\int_{t_{0}}^{t} p^{q}(s) e^{-q s} u^{q \gamma}(s-r) d s\right)^{1 / q} \\
\leq & a(t)+\left(\frac{e^{p t} \Gamma(1-(1-\beta) p)}{p^{1-(1-\beta) p}}\right)^{1 / p} \\
& \times\left(\int_{t_{0}}^{t} b^{q}(s) e^{-q s} u^{q}(s) d s\right)^{1 / q} \\
& +\left(\frac{e^{p t} \Gamma(1-(1-\beta) p)}{p^{1-(1-\beta) p}}\right)^{1 / p} \\
& \times\left(\int_{t_{0}}^{t} p^{q}(s) e^{-q s} u^{q \gamma}(s-r) d s\right)^{1 / q}, t \in I .
\end{aligned}
$$

Using Lemma 1, we obtain

$$
\begin{aligned}
u^{q}(t) \leq & 2^{q-1} a^{q}(t)+2^{2 q-2}\left(\frac{e^{p t} \Gamma(1-(1-\beta) p)}{p^{1-(1-\beta) p}}\right)^{q / p} \\
& \times \int_{t_{0}}^{t} b^{q}(s) e^{-q s} u^{q}(s) d s \\
& +2^{2 q-2}\left(\frac{e^{p t} \Gamma(1-(1-\beta) p)}{p^{1-(1-\beta) p}}\right)^{q / p} \\
& \times \int_{t_{0}}^{t} p^{q}(s) e^{-q s} u^{q \gamma}(s-r) d s, \quad t \in I . \\
\text { Let } v(t)= & {\left[e^{-t} u(t)\right]^{q} ; \text { we get } } \\
v(t) \leq & A_{2}(t)+K_{2} \int_{t_{0}}^{t} b^{q}(s) v(s) d s \\
& +K_{2} \int_{t_{0}}^{t} p^{q}(s) R_{2}(s) v^{\gamma}(s-r) d s, \quad t \in I .
\end{aligned}
$$

Using Lemma 2, we get the first inequality of (30) and the second inequality of (30) is easily obtained. The proof is complete.

For the case of $\gamma=1$, this kind of inequalities has been considered by Pachpatte [6] and the case of retarded integral inequalities also has been obtained by Ye and Gao [5, Theorem 2.5]. So, we list only a theorem using different condition and method from Pachpatte [6, Theorem 1.2.4].

Theorem 6. Let $a(t), b(t), p(t) \in C\left(I, \mathbf{R}^{+}\right)$, and $a\left(t_{0}\right)=\varphi\left(t_{0}\right)$. If $u(t) \in C\left(I, \mathbf{R}^{+}\right)$and

$$
u(t) \leq a(t)+\int_{t_{0}}^{t}(t-s)^{\beta-1}(b(s)+p(s)) u(s) d s, \quad t \in I,
$$

where $\beta>0$, then the following assertions hold.

(i) Suppose that $\beta>1 / 2$. Then

$$
u(t) \leq A_{1}^{1 / 2}(t) e^{\left(K_{1} / 2\right) \int_{t_{0}}^{t}(b(s)+p(s))^{2} d s+t}, \quad t \in I,
$$

where $A_{1}(t)$ and $K_{1}$ are defined as those in Theorem 4.

(ii) Suppose that $\beta \in(0,1 / 2], q=(1+\beta) / \beta$ and $p=1+\beta$. Then

$$
u(t) \leq A_{2}^{1 / q}(t) e^{\left(K_{2} / q\right) \int_{t_{0}}^{t}(b(s)+p(s))^{q} d s+t}, \quad t \in I,
$$

where $A_{2}(t)$ and $K_{2}$ are defined as those in Theorem 4.

Remark 7. In [6, Theorem 1.2.4], $a(t)$ is continuously differentiable, but in Theorem $6, a(t)$ is only continuous in the interval $I \subset \mathbf{R}^{+}$, so the methods of [6, Theorem 1.2.4] are invalid for Theorem 6. In [7, Theorem 1], Ye et al. also considered the similar integral inequalities using an iterative method, but we use different methods differing from the previously mentioned two papers. 


\section{Applications to FDEs}

In this section, we present applications of Theorem 4 and Theorem 5 to study certain properties of solutions of fractional differential equations.

Consider the following fractional differential equations:

$$
\begin{gathered}
{ }_{t_{0}} \mathbf{D}_{t}^{\beta} x(t)=h(t) x(t)+f(t, x), \\
{ }_{t_{0}} \mathbf{D}_{t}^{k} x\left(t_{0}\right)=b_{k}, \quad k=0,1,2, \ldots, m-1,
\end{gathered}
$$

for $t \in I, m=-[-\beta]$, where $\mathbf{D}^{\beta}$ represents the Caputo fractional derivative of order $\beta(\beta>0), h(t) \in C\left(I, \mathbf{R}^{+}\right)$, and $f(t, x) \in C(I \times \mathbf{R}, \mathbf{R})$. The corresponding Volterra fractional integral equation, see [8, Lemma 6.2], becomes

$$
\begin{aligned}
x(t)= & \sum_{k=0}^{m-1} \frac{b_{k}}{k !}\left(t-t_{0}\right)^{k}+\frac{1}{\Gamma(\beta)} \\
& \times \int_{t_{0}}^{t}(t-s)^{\beta-1}[h(s) x(s)+f(s, x(s))] d s, \quad t \in I .
\end{aligned}
$$

Theorem 8. Suppose that $|h(t) x(t)+f(t, x)| \leq b(t)|x(t)|+$ $p(t)|x(t)|^{\gamma}$, where $b(t), p(t) \in C\left(I, \mathbf{R}^{+}\right), 0<\gamma<1$ is real number. If $x(t)$ is any solution of the initial value problem (40), then the following estimations hold.

(i) Suppose that $\beta>1 / 2$. Then

$$
\begin{aligned}
&|x(t)| \leq\left[B_{1}^{1-\gamma}(t)+\frac{(1-\gamma) K_{1}}{\Gamma^{2}(\beta)}\right. \\
& \quad \times \int_{t_{0}}^{t} e^{\left((\gamma-1) K_{1} / \Gamma^{2}(\beta)\right) \int_{t_{0}}^{s} b^{2}(\sigma) d \sigma} \\
&\left.\quad \times p^{2}(s) R_{1}(s) d s\right]^{1 / 2(1-\gamma)} \\
& \quad \times e^{\left(t+\left(K_{1} / 2 \Gamma^{2}(\beta)\right) \int_{t_{0}}^{t} b^{2}(s) d s\right)}, \quad t \in I,
\end{aligned}
$$

where $B_{1}(t)=\max _{t_{0} \leq s \leq t}\left\{2 e^{-2 t}\left(\sum_{k=0}^{m-1}\left(\left|b_{k}\right| / k !\right)\left(t-t_{0}\right)^{k}\right)^{2}\right\}$.

(ii) Suppose that $\beta \in(0,1 / 2], q=(1+\beta) / \beta$, and $p=1+\beta$. Then

$$
\begin{aligned}
&|x(t)| \leq\left[B_{2}^{1-\gamma}(t)\right.+\frac{(1-\gamma) K_{2}}{\Gamma^{q}(\beta)} \\
& \quad \times \int_{t_{0}}^{t} e^{\left((\gamma-1) K_{2} / \Gamma^{q}(\beta)\right) \int_{t_{0}}^{s} b^{q}(\sigma) d \sigma} \\
&\left.\quad \times p^{q}(s) R_{2}(s) d s\right]^{1 / q(1-\gamma)} \\
& \quad \times e^{\left(t+\left(K_{2} / q \Gamma^{q}(\beta)\right) \int_{t_{0}}^{t} b^{q}(s) d s\right)}, \quad t \in I,
\end{aligned}
$$

where $B_{2}(t)=\max _{t_{0} \leq s \leq t}\left\{2^{q-1} e^{-t q}\left(\sum_{k=0}^{m-1}\left(\left|b_{k}\right| / k !\right)(t-\right.\right.$ $\left.\left.\left.t_{0}\right)^{k}\right)^{q}\right\}$. Notice that $K_{1}, K_{2}, R_{1}(s)$, and $R_{2}(s)$ are the same as those in Theorem $4, m=-[-\beta]$.
Proof. By (41), it is easy to derive that

$$
\begin{aligned}
&|x(t)| \leq \sum_{k=0}^{m-1} \frac{\left|b_{k}\right|}{k !}\left(t-t_{0}\right)^{k}+\frac{1}{\Gamma(\beta)} \\
& \quad \times \int_{t_{0}}^{t}(t-s)^{\beta-1}[b(s) x(s)+f(s, x(s))] d s \\
& \leq \sum_{k=0}^{m-1} \frac{\left|b_{k}\right|}{k !}\left(t-t_{0}\right)^{k}+\frac{1}{\Gamma(\beta)} \\
& \quad \times \int_{t_{0}}^{t}(t-s)^{\beta-1}\left[b(s)|x(s)|+p(s)|x(s)|^{\gamma}\right] d s, \\
& \quad t \in I .
\end{aligned}
$$

Using Theorem 4, we get the desired conclusion. This proves the theorem.

Considering the following fractional differential equations:

$$
{ }_{t_{0}} \mathbf{D}_{t}^{\beta} x(t)=h(t) x(t)+f(t, x(t-r)),
$$

for $t \in I=\left[t_{0}, T\right)$, with the given initial condition $x(t)=\varphi(t)$, $t \in\left[t_{0}-r, t_{0}\right], \varphi$ is a given continuously differentiable function on $t \in\left[t_{0}-r, t_{0}\right]$ up to order $m(m=-[-\beta])$. In this case, we denote $\varphi^{(k)}\left(t_{0}\right)=b_{k}, k=0,1,2, \ldots, m-1$, and $\mathbf{D}^{\beta}, \beta, h$, and $f$ are defined as those in (40).

In [8, Lemma 6.2], the initial value problem (45) is equivalent to the Volterra fractional integral equation:

$$
\begin{gathered}
x(t)=\sum_{k=0}^{m-1} \frac{b_{k}}{k !}\left(t-t_{0}\right)^{k}+\frac{1}{\Gamma(\beta)} \\
\times \int_{t_{0}}^{t}(t-s)^{\beta-1}[h(s) x(s)+f(s, x(s-r))] d s, \\
x(t)=\varphi(t), \quad t \in\left[t_{0}-r, t_{0}\right) .
\end{gathered}
$$

The next result deals with the upper bounds of solution of (45).

Theorem 9. Suppose that $|h(t) x(t)+f(t, x)| \leq b(t)|x(t)|+$ $p(t)|x(t)|^{\gamma}$, where $b(t), p(t) \in C\left(I, \mathbf{R}^{+}\right)$, and $0<\gamma<1$ is real number. If $x(t)$ is any solution of the initial value problem (46), then the following estimations hold.

(i) Suppose that $\beta>1 / 2$. Then

$$
\begin{aligned}
|x(t)| \leq[ & B_{1}^{1-\gamma}(t)+\frac{(1-\gamma) K_{1}}{\Gamma^{2}(\beta)} \\
& \quad \times \int_{t_{0}}^{t} e^{\left((\gamma-1) K_{1} / \Gamma^{2}(\beta)\right) \int_{t_{0}}^{s} b^{2}(\sigma) d \sigma} \\
& \left.\times p^{2}(s) R_{1}(s) d s\right]^{1 / 2(1-\gamma)} \\
& \times e^{\left(t+\left(K_{1} / 2 \Gamma^{2}(\beta)\right) \int_{t_{0}}^{t} b^{2}(s) d s\right)}, \quad t \in\left[t_{0}+r, T\right),
\end{aligned}
$$




$$
\begin{aligned}
|x(t)| \leq[ & B_{1}\left(t_{0}+r\right)+\frac{K_{1}}{\Gamma^{2}(\beta)} \\
& \times \int_{t_{0}}^{t_{0}+r} p^{2}(s) R_{1}(s) \varphi^{2 \gamma} \\
& \left.\quad \times(s-r) e^{-2 \gamma(s-r)} d s\right]^{1 / 2} \\
& \times e^{\left(t+\left(K_{1} / 2 \Gamma^{2}(\beta)\right) \int_{t_{0}}^{t} b^{2}(s) d s\right)}, \quad t \in\left[t_{0}, t_{0}+r\right) .
\end{aligned}
$$

(ii) Suppose that $\beta \in(0,1 / 2], q=(1+\beta) / \beta$ and $p=1+\beta$. Then

$$
\begin{aligned}
|x(t)| \leq[ & B_{2}^{1-\gamma}(t)+\frac{(1-\gamma) K_{2}}{\Gamma^{q}(\beta)} \\
& \times \int_{t_{0}}^{t} e^{\left((\gamma-1) K_{2} / \Gamma^{q}(\beta)\right) \int_{t_{0}}^{s} b^{q}(\sigma) d \sigma} \\
& \left.\times p^{q}(s) R_{2}(s) d s\right]^{1 / q(1-\gamma)} \\
& \times e^{\left(t+\left(K_{2} / q^{\Gamma^{q}}(\beta)\right) \int_{t_{0}}^{t} b^{q}(s) d s\right)}, \quad t \in\left[t_{0}+r, T\right), \\
|x(t)| \leq & {\left[B_{2}\left(t_{0}+r\right)+\frac{K_{2}}{\Gamma^{q}(\beta)}\right.} \\
& \quad \times \int_{t_{0}}^{t_{0}+r} p^{q}(s) R_{2}(s) \varphi^{q \gamma} \\
& \left.\times(s-r) e^{-q \gamma(s-r)} d s\right]^{1 / q} \\
& \times e^{\left(t+\left(K_{2} / q^{\Gamma^{q}}(\beta)\right) \int_{t_{0}}^{t} b^{q}(s) d s\right)}, \quad t \in\left[t_{0}, t_{0}+r\right) .
\end{aligned}
$$

Notice that $B_{1}(t), B_{2}(t), K_{1}, K_{2}, R_{1}(s)$, and $R_{2}(s)$ are the same as those in Theorem $8, m=-[-\beta]$.

The proof of this theorem is omitted because it is similar to that of Theorem 8 .

\section{Acknowledgments}

The authors thank the referee for his/her useful comments on this paper. This research was partially supported by the NSF of China (Grants 11171178 and 11271225), Science and Technology Project of High Schools of Shandong Province (Grant J12LI52), and program for Scientific Research Innovation Team in Colleges and Universities of Shandong Province.

\section{References}

[1] R. P. Agarwal, S. Deng, and W. Zhang, "Generalization of a retarded Gronwall-like inequality and its applications," Applied Mathematics and Computation, vol. 165, no. 3, pp. 599-612, 2005.
[2] A. A. Kilbas, H. M. Srivastava, and J. J. Trujillo, Theory and Applications of Fractional Differential Equations, vol. 204 of North-Holland Mathematics Studies, Elsevier, Amsterdam, The Netherlands, 2006.

[3] O. Lipovan, "A retarded Gronwall-like inequality and its applications," Journal of Mathematical Analysis and Applications, vol. 252, no. 1, pp. 389-401, 2000.

[4] Q.-H. Ma and J. Pečarić, "Some new explicit bounds for weakly singular integral inequalities with applications to fractional differential and integral equations," Journal of Mathematical Analysis and Applications, vol. 341, no. 2, pp. 894-905, 2008.

[5] H. Ye and J. Gao, "Henry-Gronwall type retarded integral inequalities and their applications to fractional differential equations with delay," Applied Mathematics and Computation, vol. 218, no. 8, pp. 4152-4160, 2011.

[6] B. G. Pachpatte, Integral and Finite Difference Inequalities and Applications, Elsevier, Oxford, UK, 2006.

[7] H. Ye, J. Gao, and Y. Ding, "A generalized Gronwall inequality and its application to a fractional differential equation," Journal of Mathematical Analysis and Applications, vol. 328, no. 2, pp. 1075-1081, 2007.

[8] I. Podlubny, Fractional Differential Equations, Academic Press, San Diego, Calif, USA, 1999. 


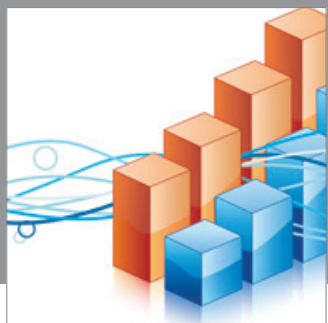

Advances in

Operations Research

mansans

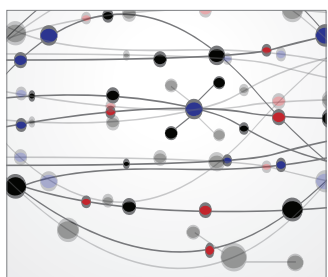

The Scientific World Journal
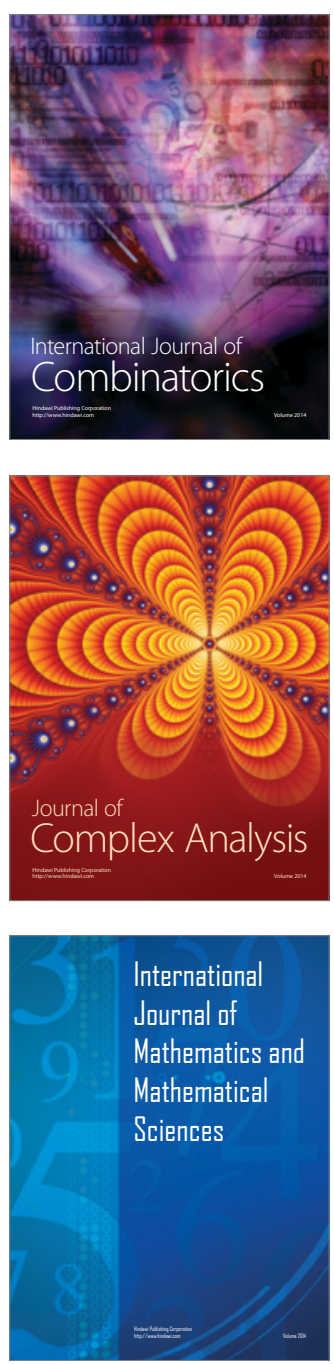
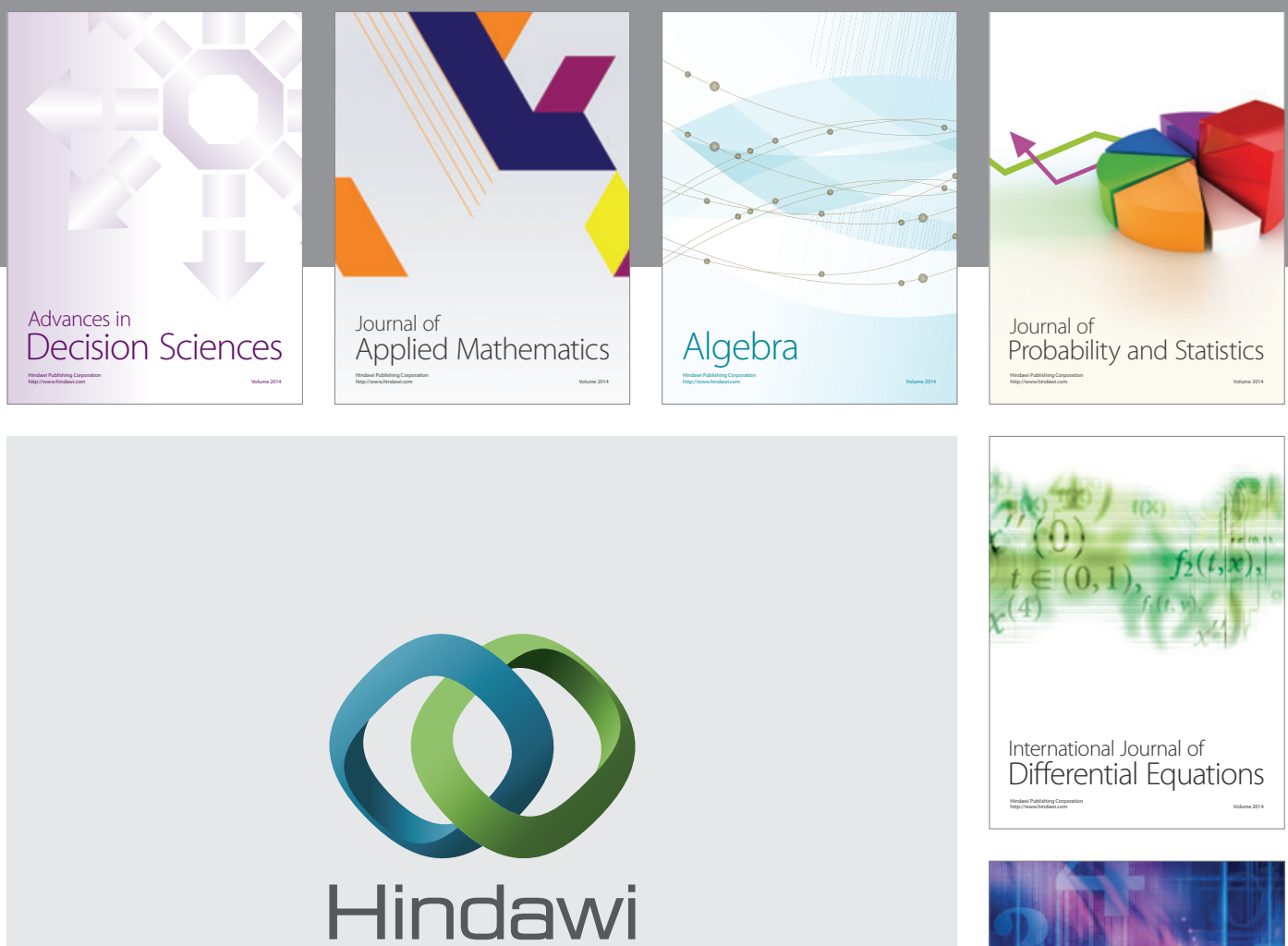

Submit your manuscripts at http://www.hindawi.com
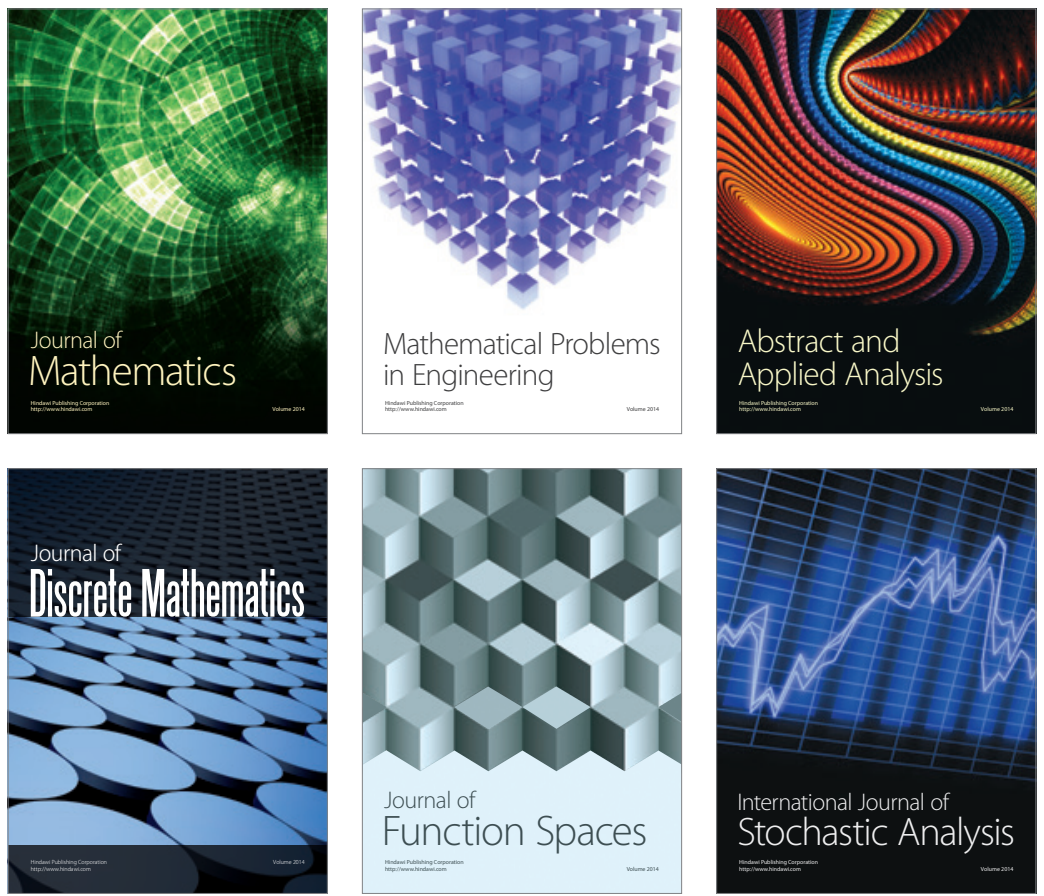

Journal of

Function Spaces

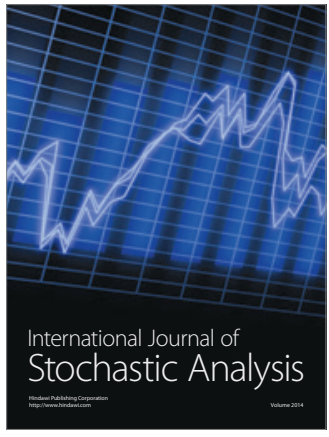

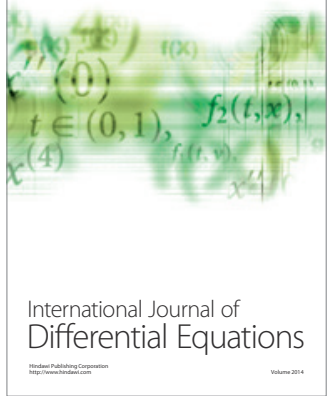
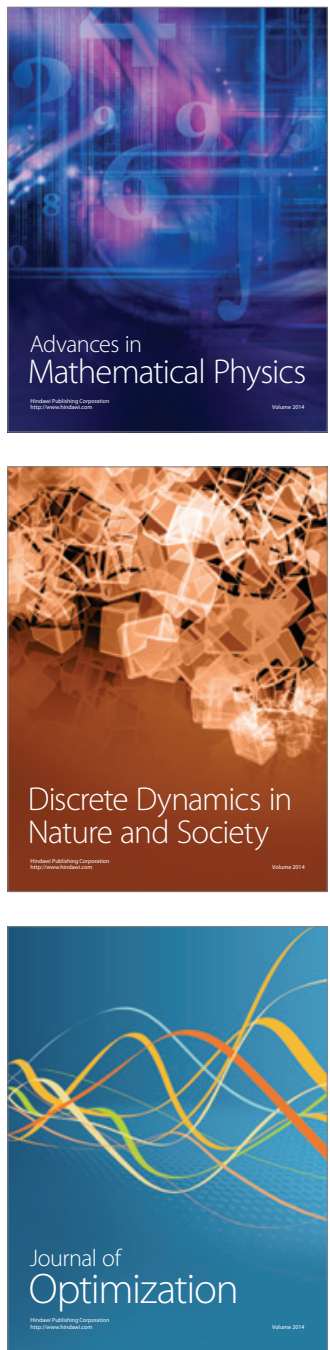